\title{
Identification of small-molecule inhibitors of Zika virus infection and induced neural cell death via a drug
} repurposing screen

\author{
Miao Xu ${ }^{1,2,16}$, Emily M Lee ${ }^{3,16}$, Zhexing Wen ${ }^{4-7,16}$, Yichen Cheng ${ }^{3}$, Wei-Kai Huang ${ }^{7,8}$, Xuyu Qian $^{7,9}$, Julia TCW $^{10}$, \\ Jennifer Kouznetsova ${ }^{1}$, Sarah C Ogden ${ }^{3}$, Christy Hammack ${ }^{3}$, Fadi Jacob ${ }^{7,11}$, Ha Nam Nguyen ${ }^{7,12}$, Misha Itkin ${ }^{1}$, \\ Catherine Hanna ${ }^{3}$, Paul Shinn ${ }^{1}$, Chase Allen ${ }^{3}$, Samuel G Michael ${ }^{1}$, Anton Simeonov ${ }^{1}$, Wenwei Huang ${ }^{1}$, \\ Kimberly M Christian ${ }^{7,12}$, Alison Goate ${ }^{10}$, Kristen J Brennand ${ }^{13}$, Ruili Huang ${ }^{1}$, Menghang Xia ${ }^{1}$, \\ Guo-li Ming ${ }^{7,9,11,12,14,15,17}$, Wei Zheng ${ }^{1,17}$, Hongjun Song ${ }^{7,9,11,12,15,17}$ \& Hengli Tang ${ }^{3,17}$
}

In response to the current global health emergency posed by the Zika virus (ZIKV) outbreak and its link to microcephaly and other neurological conditions, we performed a drug repurposing screen of $\sim 6,000$ compounds that included approved drugs, clinical trial drug candidates and pharmacologically active compounds; we identified compounds that either inhibit ZIKV infection or suppress infection-induced caspase-3 activity in different neural cells. A pan-caspase inhibitor, emricasan, inhibited ZIKV-induced increases in caspase-3 activity and protected human cortical neural progenitors in both monolayer and three-dimensional organoid cultures. Ten structurally unrelated inhibitors of cyclin-dependent kinases inhibited ZIKV replication. Niclosamide, a category B anthelmintic drug approved by the US Food and Drug Administration, also inhibited ZIKV replication. Finally, combination treatments using one compound from each category (neuroprotective and antiviral) further increased protection of human neural progenitors and astrocytes from ZIKV-induced cell death. Our results demonstrate the efficacy of this screening strategy and identify lead compounds for anti-ZIKV drug development.

ZIKV, a mosquito-borne flavivirus, has spread across the Western Hemisphere in the past year. First isolated in 1947 from a sentinel rhesus macaque in the Ziika Forest region of Uganda ${ }^{1}$, ZIKV remained in relative obscurity for many years until outbreaks occurred in the Pacific islands and then in the Americas in the past decade. A large outbreak started in Brazil in late 2014, and it is a growing public health concern $^{2}$. Active transmission has been reported in approximately 60 countries and territories globally. Most human infections are transmitted by mosquitos; however, ZIKV can also spread directly through sexual contact ${ }^{3-6}$ and vertically from mother to fetus ${ }^{7-9}$. About $20 \%$ of ZIKV-infected individuals develop symptoms, which mostly resemble those caused by other arboviruses, such as dengue viruses or chikungunya virus. Unlike these viruses, however, ZIKV causes congenital defects, including microcephaly ${ }^{10,11}$, and is associated with Guillain-Barré syndrome, meningoencephalitis and myelitis in infected adults ${ }^{8,11-14}$.

Since the recent declaration by the World Health Organization (WHO) that ZIKV is a global health concern, rapid progress has been made to understand its pathogenesis and to develop human in vitro models and animal in vivo models ${ }^{15-23}$. Following clinical observations of ZIKV in fetal brains obtained from infected women ${ }^{9,10}$, we reported that ZIKV efficiently targets human neural progenitor cells (hNPCs) and attenuates their growth ${ }^{15}$. This finding provides a potential mechanism for ZIKV-induced microcephaly as hNPCs drive the development of the cortex in the human brain. Furthermore, we and others have shown that ZIKV infection of brain organoids, which are used as three-dimen-

\footnotetext{
${ }^{1}$ National Center for Advancing Translational Sciences, National Institutes of Health, Bethesda, Maryland, USA. ${ }^{2}$ Sir Run Run Shaw Hospital, Zhejiang University School of Medicine, Hangzhou, China. ${ }^{3}$ Department of Biological Science, Florida State University, Tallahassee, Florida, USA. ${ }^{4}$ Department of Psychiatry and Behavioral Science, Emory University School of Medicine, Atlanta, Georgia, USA. ${ }^{5}$ Department of Cell Biology, Emory University School of Medicine, Atlanta, Georgia, USA. ${ }^{6}$ Department of Neurology, Emory University School of Medicine, Atlanta, Georgia, USA. ${ }^{7}$ Institute for Cell Engineering, Johns Hopkins University School of Medicine, Baltimore, Maryland, USA. ${ }^{8}$ Department of Pathology, Johns Hopkins University School of Medicine, Baltimore, Maryland, USA. ${ }^{9}$ Biomedical Engineering Graduate Program, Johns Hopkins University School of Medicine, Baltimore, Maryland, USA. ${ }^{10}$ Department of Neuroscience, Icahn School of Medicine at Mount Sinai, New York, New York, USA. ${ }^{11}$ The Solomon H. Snyder Department of Neuroscience, Johns Hopkins University School of Medicine, Baltimore, Maryland, USA. ${ }^{12}$ Department of Neurology, Johns Hopkins University School of Medicine, Baltimore, Maryland, USA. ${ }^{13}$ Department of Psychiatry, Icahn School of Medicine at Mount Sinai, New York, New York, USA. ${ }^{14}$ Department of Psychiatry and Behavioral Sciences, Johns Hopkins University School of Medicine, Baltimore, Maryland, USA. ${ }^{15}$ The Kavli Neuroscience Discovery Institute, Johns Hopkins University School of Medicine, Baltimore, Maryland, USA. 16These authors contributed equally to this work. ${ }^{17}$ These authors jointly directed this work. Correspondence should be addressed to H.S. (shongju1@jhmi.edu), G.M. (gming1@jhmi.edu), W.Z. (wzheng@mail. nih.gov) or H.T. (tang@bio.fsu.edu).
} 
a

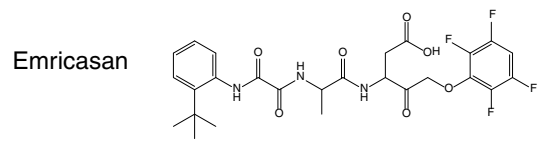
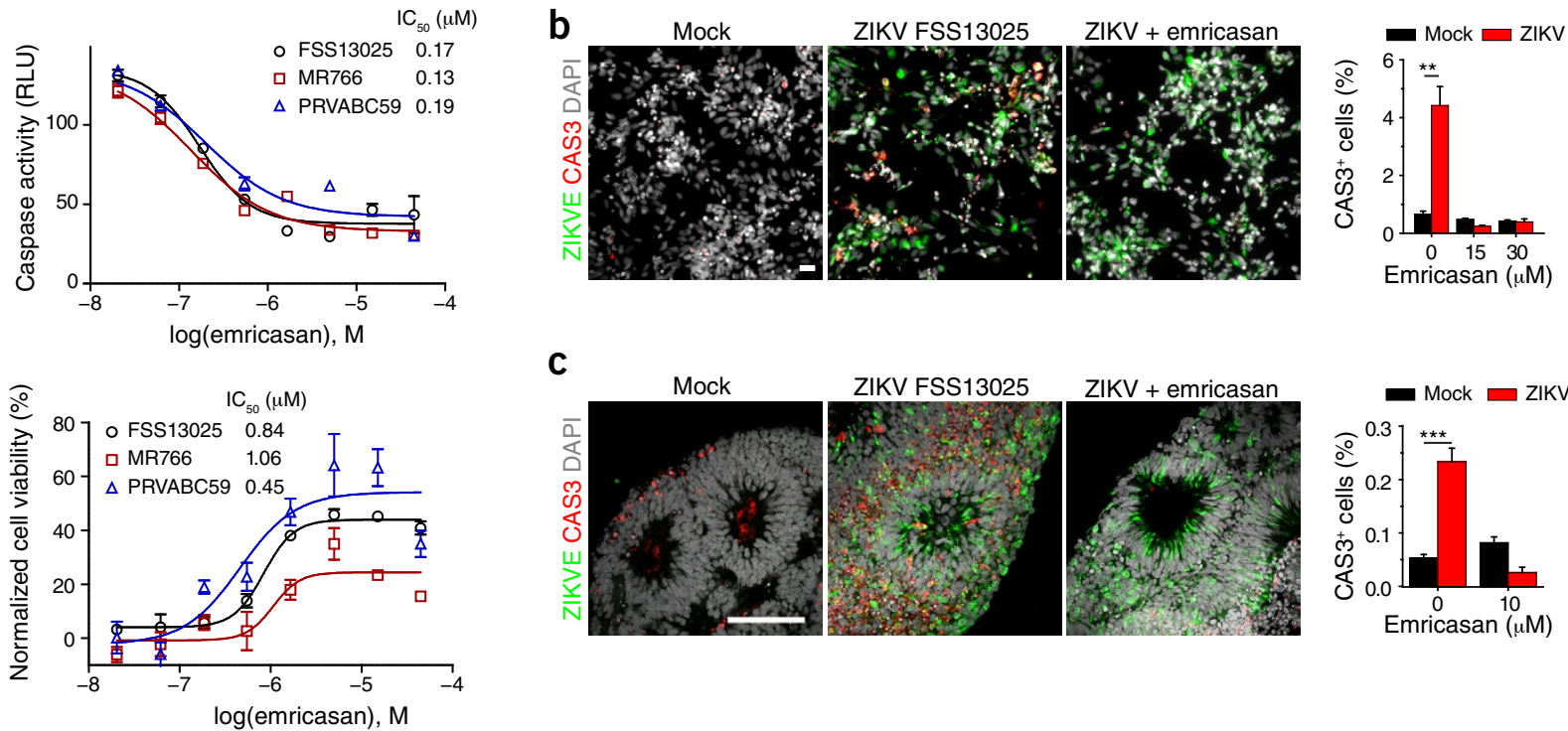

C

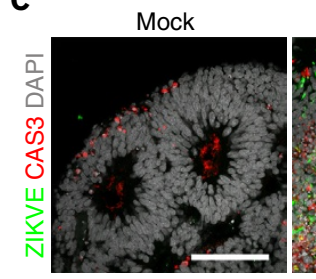

ZIKV FSS13025

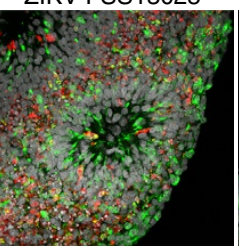

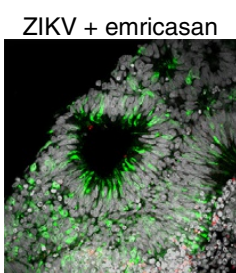

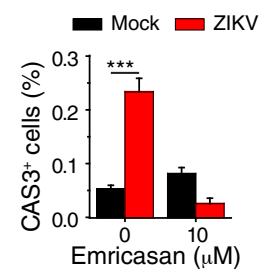

Figure 1 Emricasan suppresses cell death of ZIKV-infected human astrocytes and hNPCs in 2D monolayer cultures and in 3D brain organoids. (a) Top, chemical structure of emricasan. Bottom, dose-response curves showing the effect of emricasan treatment on caspase activity (middle) and cell viability (bottom) in SNB-19 glioblastoma cells exposed to three different ZIKV strains. Values represent mean \pm s.d. ( $n=3$ cultures). Curves represent best fits for calculating the $\mathrm{IC}_{50}$ values, and the insets in each graph report the calculated $\mathrm{IC}_{50}$ value against each strain. RLU, relative luminescence units. (b) Left, representative images of 2D monolayer cultures of forebrain-specific hNPCs immunostained for ZIKV protein (ZIKVE; green), cleaved caspase-3 (CAS3; red) and nuclei (with DAPI; gray) $72 \mathrm{~h}$ after ZIKV FSS-13025 exposure (with or without emricasan treatment) or mock infections (multiplicity of infection (MOI) $=0.04-0.08$ ). Scale bar, $20 \mu \mathrm{m}$. Right, quantification of the percentage of CAS3 ${ }^{+}$cells, relative to the total number of DAPI-stained cells, in mock or ZIKVinfected hNPCs cultures treated with the indicated concentrations of emricasan ( $n=3$ cultures). Values represent mean \pm s.e.m. ${ }^{* *} P<0.01$; by one-way ANOVA. (c) Left, representative images of 3D human forebrain-specific organoid cultures (28 d in vitro) immunostained for ZIKVE (green), CAS3 (red) and nuclei (with DAPI; gray), $10 \mathrm{~d}$ after ZIKV FSS-13025 exposure. Scale bar, $100 \mu \mathrm{m}$. Right, quantification of the percentage of CAS3 ${ }^{+}$cells, relative to the total number of DAPI-stained cells, in the ventricular zone ( $n=6$ organoids). Values represent mean \pm s.e.m. ${ }^{* * *} P<0.001$; by one-way ANOVA.

sional (3D) cellular models of early human brain development, leads to reduced thickness of hNPC and neuronal layers, and an overall reduction in organoid size $\mathrm{s}^{16,17,20,24}$, observations that are again consistent with features of microcephaly. These results have also been recapitulated in mouse models ${ }^{20,21,23}$. Despite these advancements in understanding how ZIKV causes developmental abnormalities and in preclinical studies that are underway to develop vaccines ${ }^{25,26}$, there is currently no drug approved to treat or prevent ZIKV infection.

Drug repurposing screens have recently emerged as an alternative approach to accelerate drug development ${ }^{27,28}$. Following a repurposing phenotypic screen, new indications for existing drugs may be rapidly identified, and clinical trials can be carried out quickly, which is especially critical for rapidly spreading infectious diseases. For example, recent drug repurposing screens have led to discoveries of potential new candidate therapies for Ebola virus disease ${ }^{29,30}$, giardiasis ${ }^{31}$, Entamoeba histolytica infection ${ }^{32}$, malaria gametocytes ${ }^{33}$, Exserohilum rostratum infection ${ }^{34}$, hepatitis $\mathrm{C}$ virus (HCV) infection ${ }^{35}$ and, very recently, ZIKV infection ${ }^{36}$. On the basis of our previous finding that ZIKV infection of hNPCs results in an increase in caspase- 3 activation, followed by cell death ${ }^{15}$, we designed a compound-screening approach using caspase- 3 activity as the primary screening assay, with a secondary cell viability assay for confirmation (Supplementary Fig. 1a). We identified two classes of effective compounds that are capable of protecting neural cells from ZIKV-induced cell death, one consisting of compounds that are antiviral in nature and the other of compounds that are neuroprotective in nature.

\section{RESULTS}

Development of high-throughput compound-screening approaches We first quantified caspase- 3 activity and cell viability in hNPCs and astrocytes that were derived from human induced pluripotent stem cells (iPSCs), as well as in glioblastoma SNB-19 cells, after ZIKV infection in a 1,536-well plate format (Supplementary Tables 1 and 2). The prototypic ZIKV strain, MR766, was used in the primary screen because it produced the strongest cell death signal in cell culture experiments. The signal-tobasal $(\mathrm{S} / \mathrm{B})$ ratios and the coefficients of variation $(\mathrm{CV})$ obtained in the caspase-3 activity assay after a 6-h ZIKV exposure were 2.1-fold and 7.0\% for hNPCs, 7.0 -fold and 5.9\% for SNB-19 cells and 11.0 -fold and $9.1 \%$ for astrocytes (Supplementary Fig. 1b). The Z' factors, which are a measure of statistical effect size and an index for assay quality control ${ }^{37}$, for hNPCs, SNB-19 and astrocytes were $0.20,0.68$ and 0.72 , respectively. Because a $Z^{\prime}$ factor $>0.5$ indicates a robust screening assay ${ }^{37}$, the caspase assay using SNB-19 cells or astrocytes is suitable for high-throughput screening.

To measure cell viability, we performed an ATP-content assay following ZIKV infection for $3 \mathrm{~d}$ (Supplementary Table 2). Cell viability was reduced by $39 \%, 82 \%$ and $69 \%$ in hNPCs, SNB-19 cells and human astrocytes, respectively (Supplementary Fig. 1c). The Z' factors in these three cell types were $0.06,0.37$ and 0.32 , respectively. These results indicated that measuring caspase- 3 activity is a better assay for high-throughput compound screening than the cell-viability assay.

\section{High-throughput screen of compound collections}

We carried out a screening campaign, using the caspase- 3 activity 

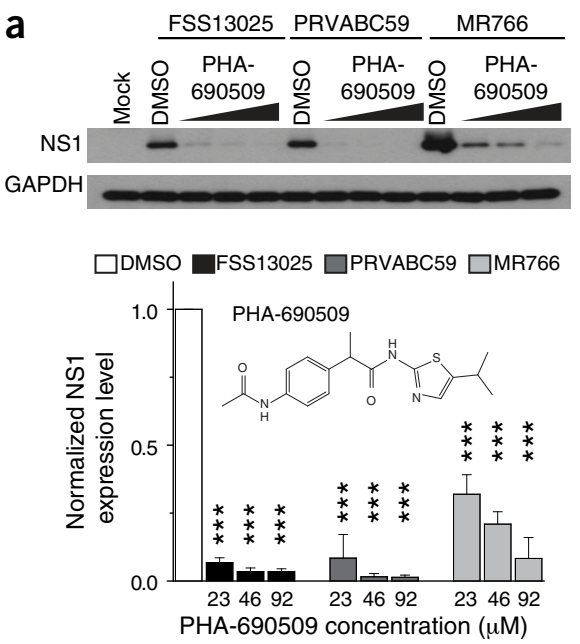

d

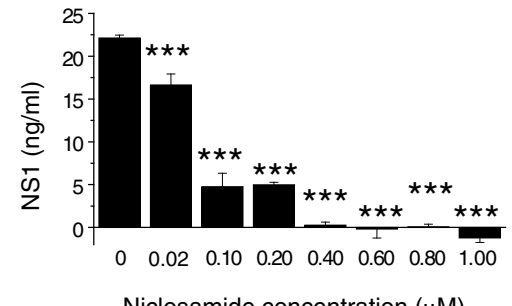

b b

NS1

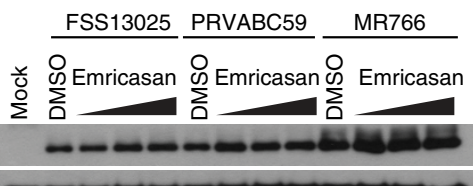
GAPDH

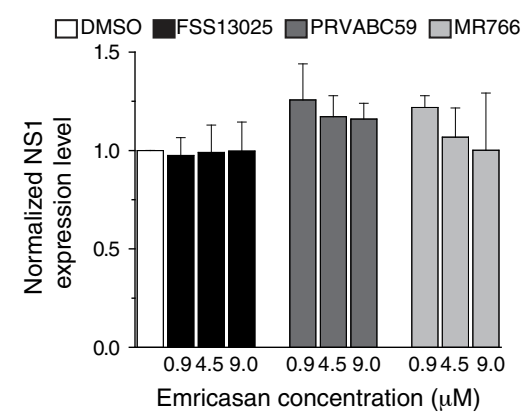

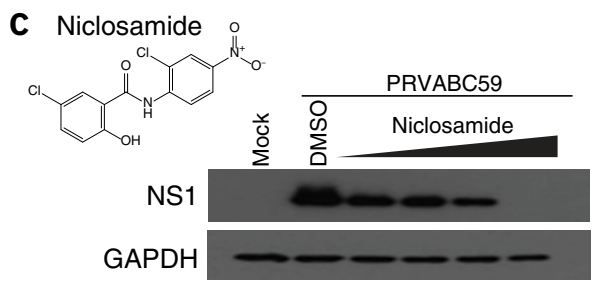

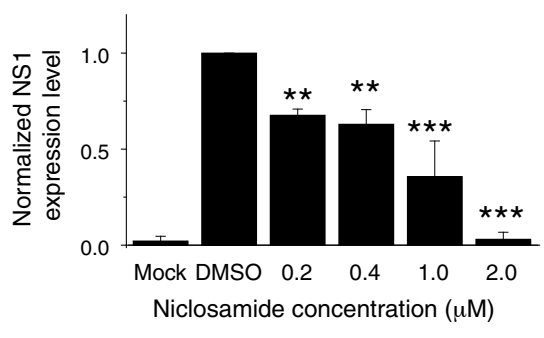

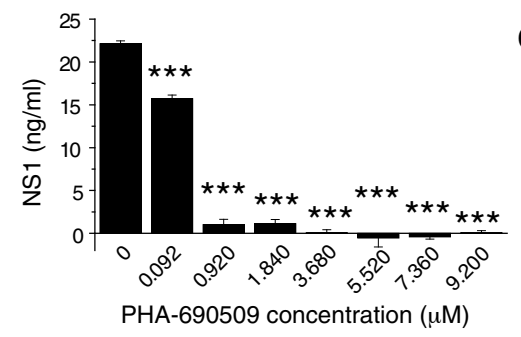
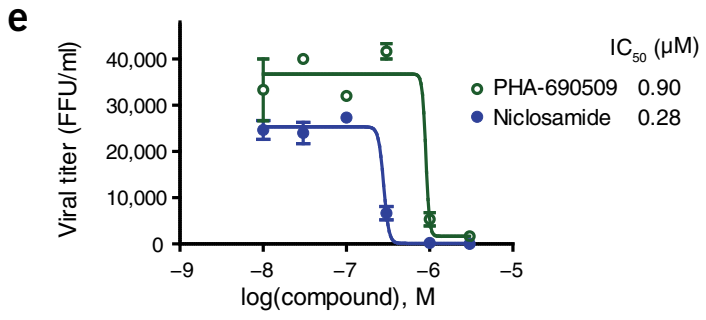

Figure 2 Identification of PHA-690509 and niclosamide as antiviral compounds. (a) Top, representative western blot analysis for anti-ZIKV activity after treatment of SNB-19 cells with varying concentrations of PHA-690509. Glioblastoma SNB-19 cells were treated with the indicated compound for $1 \mathrm{~h}$ before inoculation with ZIKV FSS-13025, PRVABC59 or MR766 (MOI = 1), and cells were harvested $24 \mathrm{~h}$ after infection for western blot analysis. Bottom, quantification of NS1 protein band intensities, relative to those of GAPDH ( $n=3$ cultures). Data were normalized to that for DMSO-treated cells. Values represent mean \pm s.d. ${ }^{* * *} P<0.001$; by one-way ANOVA in comparison to DMSO-treated cells. Inset shows chemical structure of PHA-690509. (b) Top, representative western blot images of titration for anti-ZIKV activity after treatment of SNB-19 cells with emricasan. Bottom, quantification as in a ( $n=3$ cultures). Values represent mean \pm s.d. $P>0.1$; by one-way ANOVA, as compared to DMSO-treated cells. (c) Top, chemical structure of niclosamide and representative western blot images for anti-ZIKV activity after treatment of SNB-19 cells with varying concentrations of niclosamide, using the PRVABC59 strain $(\mathrm{MOI}=1)$. Bottom, quantification as in a ( $n=3$ cultures). Values represent mean $\pm \mathrm{s}$.d. ${ }^{* *} P<0.01$; ${ }^{* * *} P<0.001$; by one-way ANOVA, as compared to DMSO-treated cells. (d) Summary of ELISA quantifications of secreted ZIKV NS1 protein in the medium of infected cells after treatment of cells with different doses of niclosamide (left) or PHA-690509 (right) for $24 \mathrm{~h}$ ( $n=3$ cultures). Values represent mean \pm s.d. *** $P<0.001$; by one-way ANOVA, as compared to the $0 \mu \mathrm{M}$ group. (e) Summary of titration of niclosamide and PHA-690509 on ZIKV production. SNB-19 cells were treated with each indicated compound at increasing concentrations for $1 \mathrm{~h}$ before infection with PRVABC59 at a MOI = 0.5 ( $n=3$ cultures). Cell culture supernatant was collected $24 \mathrm{~h}$ after infection, and infectious virions were quantified for focus-forming units (FFU) using Vero cells. Data represent mean \pm s.d. Curves represent best fits for calculating $\mathrm{IC}_{50}$ values, and the insets report the calculated $\mathrm{IC}_{50}$ value for each compound.

assay and SNB-19 cells, with the Library of Pharmacologically Active Compounds (LOPAC, 1,280 compounds), the National Center for Advancing Translational Sciences (NCATS ) Pharmaceutical Collection of approved drugs (2,816 compounds) and a collection of clinical candidate compounds (2,000 compounds). Primary hits included a total of 116 compounds that suppressed ZIKV-induced caspase-3 activity in SNB-19 cells. We also carried out an independent primary screen in hNPCs using the same libraries. This second screen resulted in 173 primary hits that included all 116 compounds from the initial caspase-3 screen in SNB-19 cells. All of the results from the primary screen with the approved drug collections, and hit confirmation, were deposited into the open-access PubChem database (https://pubchem.ncbi.nlm. nih.gov/).

Next, the activity of these primary hits from the caspase- 3 activity assay was re-evaluated in ZIKV-infected SNB-19 cells, hNPCs and astrocytes, and, notably, was performed in parallel with the compound cytotoxicity assay (Supplementary Fig. 1d,e and Supplementary Table 3). Cytotoxic compounds were then eliminated from the confirmedhit list. Consistent with the screening design, we identified compounds that reduced virally induced caspase activation and apoptosis by either directly preventing ZIKV-induced cell death or suppressing ZIKV replication (Supplementary Table 4).

\section{Protection from ZIKV-induced cell death by emricasan}

Emricasan, a pan-caspase inhibitor, was identified as the most potent anti-cell-death compound, with half-maximal inhibitory concentration $\left(\mathrm{IC}_{50}\right.$ ) values of $0.13-0.9 \mu \mathrm{M}$ in both caspase activity and cell viability assays for SNB-19 cells against three ZIKV strains: MR766 (1947 Ugandan strain), FSS13025 (2010 Cambodian strain) and PRVABC59 (2015 Puerto Rican strain) (Fig. 1a). It was also effective for all three cell types tested (Supplementary Fig. If and Supplementary Table 5). In addition, emricasan treatment reduced the number of active (cleaved) caspase-3-expressing forebrain-specific hNPCs after infection with FSS13025, in both monolayer and 3D organoid cultures (Fig. 1b,c). Emricasan treatment of ZIKV-exposed brain organoids did not seem to affect hNPC proliferation, as compared to the mock-treated organoids, when evaluated by phosphohistone3 (PH3) expression (106 $\pm 10 \% ; n=8 ; P=0.7$; one-way analysis of variance (ANOVA)). Notably, ZIKV antigen persisted in both $2 \mathrm{D}$ and $3 \mathrm{D}$ cultures after emricasan treatment (Fig. 1b,c). Therefore, emricasan shows neuroprotective activity for hNPCs but does not suppress ZIKV replication. 
a

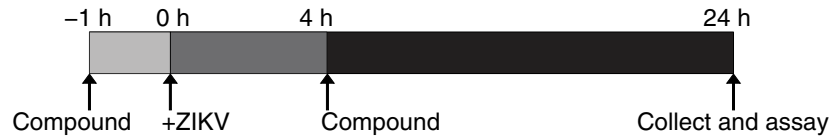

C

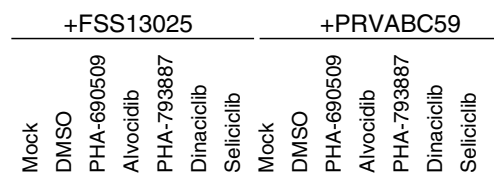

b

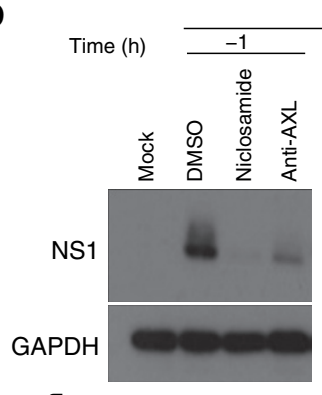

+ PRVA
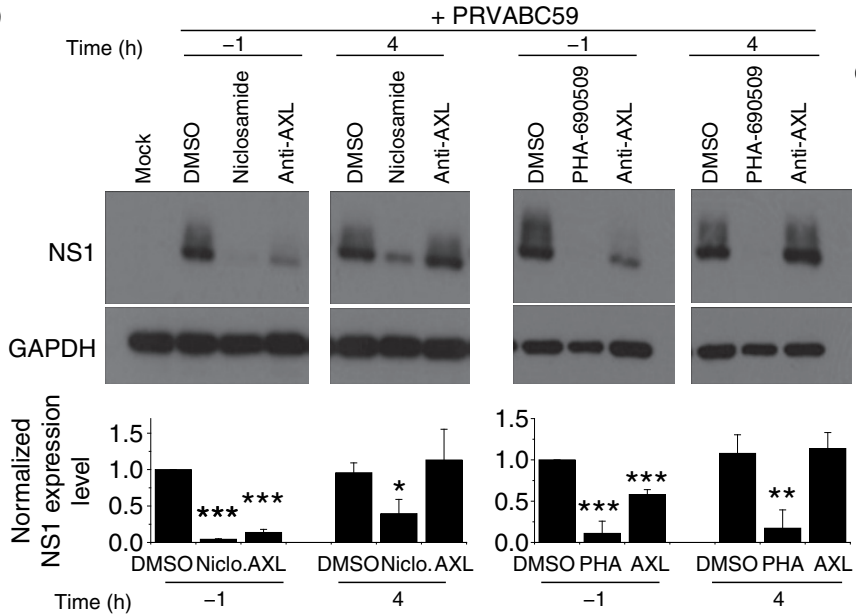

NS1

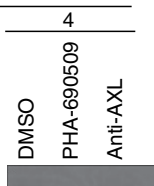

GAPDH

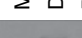

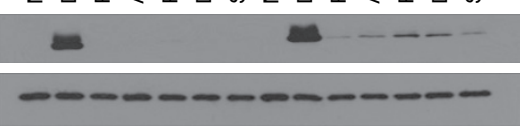

d
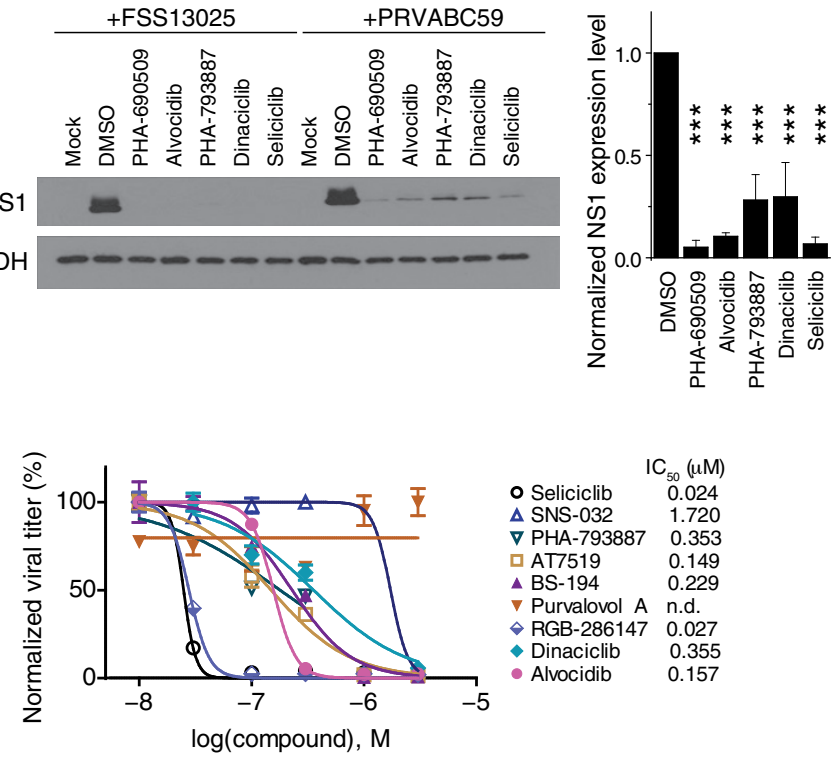

Figure 3 Inhibition of ZIKV infection by niclosamide and PHA-690509 at a post-entry step and blockade of ZIKV infection by additional treatment with CDKis. (a) Schematic illustration of a time-of-addition experiment for treatment with niclosamide and PHA-690509. (b) Top, representative western blot images of SNB-19 cells after treatment with $10 \mu \mathrm{g} / \mathrm{ml}$ AXL-specific antibody, $2 \mu \mathrm{M}$ niclosamide (Niclo.) or $92 \mu \mathrm{M}$ PHA-690509 for $1 \mathrm{~h}$ before or $4 \mathrm{~h}$ after infection with PRVABC59 $(\mathrm{MOI}=1)$. Bottom, quantification of NS1 protein band intensities, relative to those for GAPDH ( $n=3$ cultures). Data were normalized to those obtained for DMSO-treated cells. Values represent mean \pm s.d. ${ }^{*} P<0.05$; ${ }^{* *} P<0.01$; ${ }^{* * *} P<0.001$; by one-way ANOVA, as compared to DMSO-treated cells. (c) Inhibition of ZIKV infection in CDKi-treated cells. Left, representative western blot images of SNB-19 cells treated with $92 \mu \mathrm{M}$ of the indicated compound for $1 \mathrm{~h}$ before infection with FSS13025 or PRVABC59 (MOI = 1). Right, quantification as in b. Values represent mean \pm s.d. ( $n=3$ cultures; *** $P<0.01$; one-way ANOVA for comparison with the DMSO treatment). (d) Effect of various CDKis on ZIKV production (as in Fig. 2e) ( $n=3$ cultures). All data were normalized to that for $0 \mu \mathrm{M}$ for each compound. Data represent mean \pm s.d. Curves represent best fits for calculating $I C_{50}$ values (listed to the right).

\section{Identification of antiviral compounds against ZIKV}

Using expression of ZIKV protein NS1 as a read-out to screen anti-ZIKV activity, we identified two compounds from the primary hit list that substantially inhibited ZIKV infection of SNB-19 cells (Supplementary Fig. 2a). The first was niclosamide, a US Food and Drug Administration (FDA)-approved drug for treating worm infections in both humans and domestic livestock; the other was PHA-690509, an investigational compound that functions as a cyclin-dependent kinase inhibitor (CDKi). Both compounds inhibited replication of all three strains of ZIKV, as measured by NS1 expression, in a dose-dependent manner (Fig. 2a-d and Supplementary Fig. 2b,c).

We further validated the antiviral activity of these two compounds using independent approaches. First, measurement of intracellular ZIKV RNA levels showed $\mathrm{IC}_{50}$ values of $1.72 \mu \mathrm{M}$ and $0.37 \mu \mathrm{M}$ for PHA-690509 and niclosamide, respectively (Supplementary Fig. 2d,e). Second, both compounds suppressed production of infectious ZIKV particles at submicromolar concentrations (Fig. 2e). To investigate the underlying cellular mechanism, we performed time-of-addition experiments in SNB-19 cells (Fig. 3a). Both compounds effectively inhibited ZIKV infection when added either $1 \mathrm{~h}$ before or $4 \mathrm{~h}$ after virus inoculation (Fig. 3b). In contrast, a monoclonal antibody to AXL, a putative ZIKV entry factor ${ }^{38,39}$, was only effective when added before inoculation (Fig. 3b). Furthermore, the reduction of ZIKV RNA by treatment with these compounds was apparent only after the entry phase (0-4 h after infection) and correlated with the replication phase (4-24h) of the infection cycle (Supplementary Fig. 3). Taken together, these results indicate that niclosamide and PHA-690509 inhibit ZIKV infection at a post-entry stage, probably at the viral RNA replication step.

Pharmacological CDKis have been shown to inhibit replication of diverse viruses in culture, including herpes viruses and $\mathrm{HIV}^{40-45}$, and depletion of CDK9 impairs influenza A viral replication ${ }^{46}$. The possibility of ZIKV inhibition by a CDKi is particularly intriguing, given our recent observation that ZIKV infection and cell cycle regulation are intimately connected ${ }^{15}$. We therefore tested 27 additional structurally distinct CDKis for inhibition of ZIKV infection (Supplementary Table 6). We identified nine effective compounds using SNB-19 cells (Fig. 3c,d and Supplementary Fig. 4a), whereas four non-CDK-kinase inhibitors showed minimal anti-ZIKV activity (Supplementary Fig. 4b). Analyses of ZIKV production showed $\mathrm{IC}_{50}$ values at submicromolar concentrations for nine of the ten CDKis tested, with seliciclib and RGB-286147 at $24 \mathrm{nM}$ and $27 \mathrm{nM}$, respectively (Fig. 3d).

Using the clinical isolate from the 2015 Puerto Rico Zika outbreak, PRVABC59, we next examined the effectiveness of the identified compounds in hNPCs and astrocytes, both of which are target cells for ZIKV infection in the fetal brain ${ }^{9}$. Niclosamide and PHA690509 treatments inhibited ZIKV infection and production in these central nervous system cells (Fig. 4a-c). ZIKV targets astrocytes in the adult mouse brain ${ }^{47}$. Analysis of ZIKV production after infection of human astrocytes showed $\mathrm{IC}_{50}$ values of $\sim 0.2 \mu \mathrm{M}$ for both niclosamide and PHA-690509 (Fig. 4c). Quantification of astrocyte viability showed minimal toxicity for niclosamide, PHA-690509 and four CDKis at levels $<3 \mu \mathrm{M}$ (Supplementary Fig. 5a). Given the critical role of CDKs in cell cycle regulation, we examined the effect of PHA-690509 and seliciclib, the most potent CDKi for ZIKV inhibition (Fig. 3d), on hNPC proliferation. ZIKV infection led to a drastic reduction in hNPC proliferation, which was partially rescued by treatment with either compound (Supplementary Fig. 5b). Furthermore, treatment with PHA-690509 (1 $\mu \mathrm{M})$ or seliciclib $(5 \mu \mathrm{M})$ alone had minimal effect on hNPC proliferation in brain organoids (Supplementary Fig. 5c). 
a
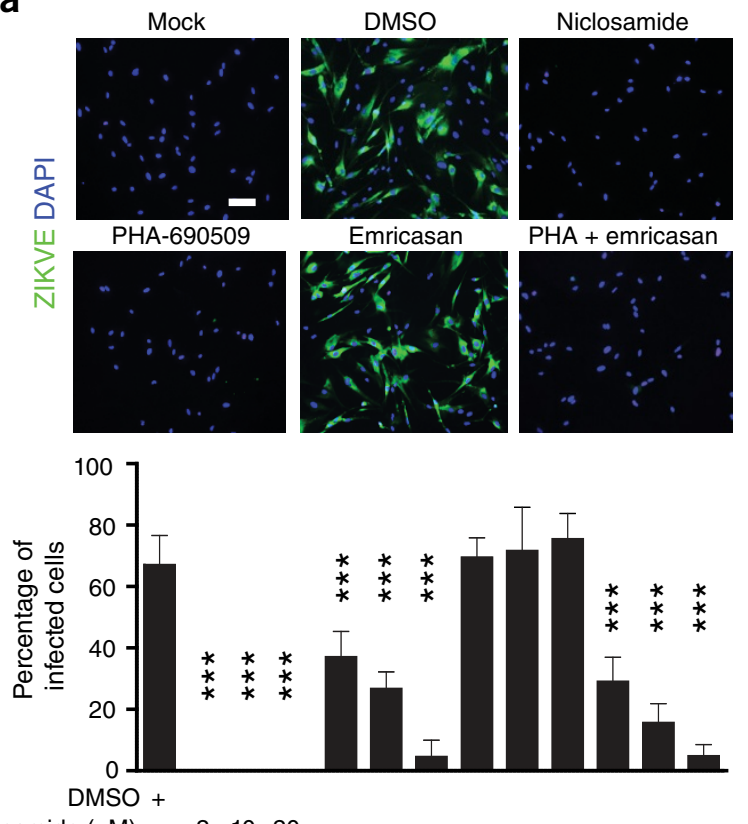

Niclosamide $(\mu \mathrm{M})$

PHA-690509 $(\mu \mathrm{M})$

Emricasan $(\mu \mathrm{M})$
$2 \quad 10 \quad 20$

$9 \quad 46 \quad 92$
9 b
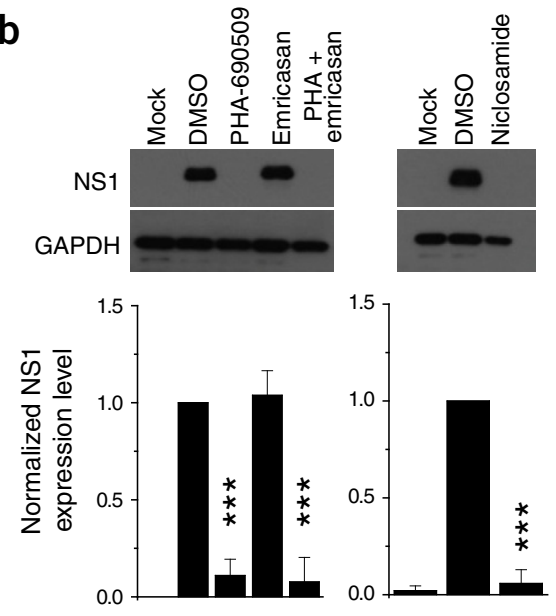

C

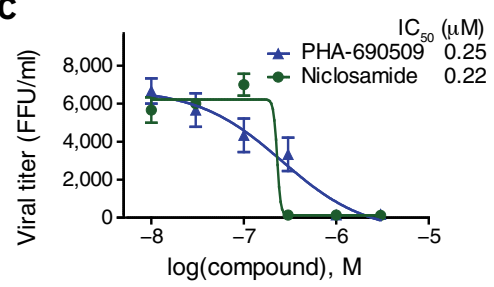

d

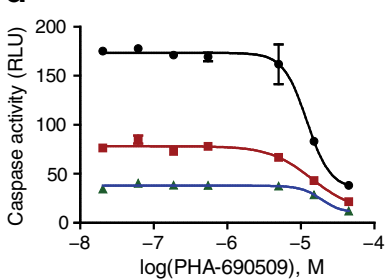

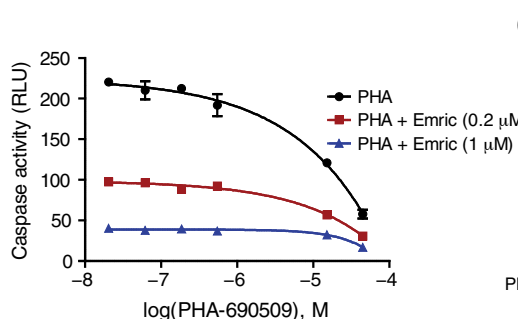

e

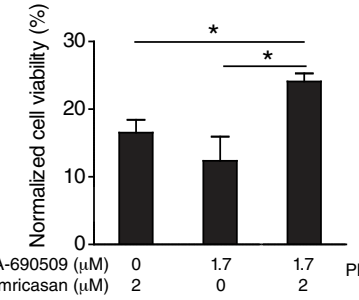

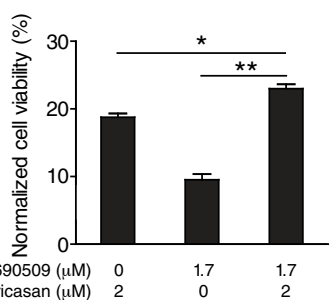

Figure 4 Niclosamide and PHA-690509 inhibit ZIKV infection in human astrocytes and forebrain-specific hNPCs. (a) Top, representative immunostained images of astrocytes that were mock-infected, treated with DMSO or treated with $2 \mu \mathrm{M}$ niclosamide, $92 \mu \mathrm{M}$ PHA-690509, $9 \mu \mathrm{M}$ emricasan, or a combination of $92 \mu \mathrm{M}$ PHA-690509 and $9 \mu \mathrm{M}$ emricasan for $1 \mathrm{~h}$ before infection with PRVABC59 $(\mathrm{MOI}=0.5)$. Cells were fixed $24 \mathrm{~h}$ after infection and stained for ZIKVE (green) and nuclei (with DAPI; blue). Scale bar, $50 \mu \mathrm{m}$. Bottom, quantification of the percentage of ZIKV-infected astrocytes, relative to the number of DAPIstained cells ( $n=6$ cultures). Values represent mean \pm s.d. ${ }^{* * *} P<0.001$; by one-way ANOVA, as compared to DMSO-treated cells. (b) Top, representative western blot images of hNPCs that were treated with the indicated compounds and infected with PRVABC59 at a MOI of 0.1 . Cells were analyzed $48 \mathrm{~h}$ after infection. Bottom, quantification of NS1 protein band intensities, relative to those of GAPDH ( $n=3$ cultures). Data were normalized to those for DMSO-treated cells. Values represent mean \pm s.d. ${ }^{* * *} P<0.01$; by one-way ANOVA, as compared to DMSO-treated cells. (c) Virus production from compound-treated iPSCderived human astrocytes (as in Fig. 2e) ( $n=3$ cultures). Data represent mean \pm s.d. (d,e) Summary of the additive effects of treatment with a combination of emricasan and PHA-690509 on caspase-3 activity (d), as well as on improving cell viability as measured by ATP production (e), in human astrocytes infected with the FSS13025 (left) or MR766 (right) strain of ZIKV $(n=3)$. Values represent mean \pm s.d. ${ }^{*} P<0.05$; ${ }^{* *} P<0.01$; by one-way ANOVA.

\section{Benefit of combining neuroprotective and antiviral compounds}

Finally we explored combining these two categories of compounds (neuroprotective and antiviral). The two-drug combination of emricasan and PHA-690509 showed an additive effect in inhibiting caspase-3 activity in SNB-19 cells (Fig. 4d). A similar additive effect was found to preserve astrocyte viability after ZIKV infection (Fig. 4). Notably, emricasan did not interfere with PHA-690509's ability to inhibit ZIKV infection in the combination treatment (Fig. $\mathbf{4 b}$ and Supplementary Fig. 6a-c).

We also tested the effect of sequential treatment with the two categories of compounds. We found that treatment of PRVABC59-infected hNPCs with emricasan for $72 \mathrm{~h}$ followed by niclosamide treatment for $48 \mathrm{~h}$ led to the recovery of ZIKV-negative hNPCs, suggesting a beneficial effect of 'buying time' by inhibiting apoptosis to allow the infected cells to recover (Supplementary Fig. 6d).

\section{DISCUSSION}

Here we developed two ultra-high-throughput assays, using human neural cells, to measure ZIKV-induced caspase- 3 activity and cell viability, and we screened over 6,000 approved drugs and drug candidate compounds. Our efforts so far have led to the identification of small molecules that either protect against cell death in multiple neural cell types or suppress ZIKV replication.

Emricasan, also called IDN-6556 or PF-03491390, is an inhibitor of activated caspases, and it has sub- to nanomolar activity in vitro ${ }^{48,49}$. Emricasan is currently being evaluated in phase 2 clinical trials for the reduction of hepatic injury and liver fibrosis caused by chronic HCV infection ${ }^{50,51}$. Emricasan was well tolerated in human trials, and there were no significant adverse events ${ }^{51}$. It was reported that overall and maximum concentrations of emricasan (orally, twice daily, $\times 4 \mathrm{~d}$ ) in human blood were $1.90 \mu \mathrm{g} / \mathrm{ml}(3.35 \mu \mathrm{M})$ and $2.36 \mu \mathrm{g} / \mathrm{ml}(4.15 \mu \mathrm{M})$, 
respectively. Therefore, the reported human plasma concentration of emricasan is about tenfold higher than the $\mathrm{IC}_{50}$ for inhibition of increased caspase- 3 activity and cell death caused by ZIKV infection in vitro. Future animal studies will be critical to evaluate the efficacy of emricasan in vivo. Whether it is safe to use emricasan during pregnancy for ZIKV infection in humans will need to be evaluated in preclinical toxicology studies and in clinical trials.

Ten structurally unrelated CDKis inhibit ZIKV replication and production, supporting a role for CDKs in the life cycle of ZIKV in human cells. Because flaviviruses are not known to encode any CDKs, these results suggest that one or more cellular CDKs in the host may be important for ZIKV replication. Further studies on target identification, including targeted kinome siRNA or CRISPR screens, may reveal additional insights into the mechanism of action for these inhibitors. Although many CDKis are being evaluated in clinical trials for various cancers, cystic fibrosis and Cushings disease, these compounds may not be suitable for pregnant women because of potentially hazardous effects on the fetus ${ }^{52}$. In our in vitro analyses, treatment with PHA-690509 or seliciclib partially rescued the ZIKV-induced reduction of hNPC proliferation, and the treatment with either compound in the absence of ZIKV infection showed a minimal effect on hNPC proliferation in brain organoid cultures, which model early human brain development in vitro (Supplementary Fig. 5c,d). Future studies in animal models, including both rodents and primates, are critical to test the efficacy and toxicity of identified compounds in vivo. Notably, these CDKis and their derivatives may be useful for treating nonpregnant humans who face an increased risk of Guillain-Barré syndrome and other conditions following ZIKV infection. For example, viral RNA and infectious virus have been detected in the semen of men months after acute symptoms have resolved $^{53}$. In addition, these CDKis will be valuable chemical tools in probing the roles of specific types of CDKs in ZIKV infection, which could serve as new targets for anti-ZIKV drug development. The structural diversity of effective CDKis also provides multiple scaffolds for lead-compound optimization using medicinal chemistry.

Niclosamide is an FDA-approved drug (trade name Niclocide) that has been used in humans to treat worm infections for nearly 50 years, and it is well tolerated ${ }^{54,55}$. It is known to inhibit several viruses in culture systems, including the Japanese encephalitis flavivirus ${ }^{56-58}$. Its broad antiviral activity has been attributed to its ability to neutralize endolysosomal $\mathrm{pH}$ and interfere with $\mathrm{pH}$-dependent membrane fusion $^{57}$, which is an essential step in the common virus entry pathway. Our time-of-addition and time-course analyses, however, suggest that inhibition by niclosamide occurs at a post-entry step, such as replication. Future molecular studies of its mechanism of action may provide additional targets for drug development. Niclosamide is a category B drug, which indicates that no risk to fetuses has been found in animal studies. It has low toxicity in mammals with an oral median lethal dose $\left(\mathrm{LD}_{50}\right)$ value of $5,000 \mathrm{mg}$ per $\mathrm{kg}$ body weight $(\mathrm{mg} / \mathrm{kg})$ in rats ${ }^{59}$. The potency of niclosamide on inhibition of ZIKV replication is in the submicromolar range, whereas clinically it can be delivered at micromolar levels ${ }^{59}$. Additionally, a pro-drug formulation may further increase the bio-availability of this compound, which is currently indicated for treating intestinal parasites. The WHO recommends that niclosamide may be used during pregnancy because it has not been shown to be mutagenic, teratogenic or embryotoxic (http://apps.who.int/medicinedocs/en/d/ Jh2922e/3.1.3.html). The US Centers for Disease Control and Prevention (CDC) concurs with the WHO recommendation and further recommends that "for individual patients in clinical settings, the risk of treatment (with Niclosamide) in pregnant women who are known to have an infection needs to be balanced with the risk of disease progression in the absence of treatment" (http://www.cdc.gov/parasites/hymenolepis/ health_professionals/). Independently of evaluating the potential benefits and risks for pregnant women, niclosamide could be used to reduce viral load in infected men and nonpregnant women, which could reduce transmission and potentially prevent Guillain-Barré syndrome and other ZIKV-related complications in humans.

Despite rapid progress in the preclinical development of anti-ZIKV vaccines ${ }^{25,26}$, testing the safety and efficacy of vaccines in humans can take a substantial amount of time. Effective countermeasures against ZIKV, including small-molecule therapeutics, are also urgently needed. Our findings and the tools provided here should significantly advance current ZIKV research and have an immediate effect on the development of anti-ZIKV therapeutics. Furthermore, our findings could have implications for combating infections by other arboviruses, such as dengue viruses, chikungunya virus and West Nile virus, many of which can cause devastating illness.

\section{METHODS}

Methods and any associated references are available in the online version of the paper.

Accession codes. PubChem Bioassay Database: all of the results from the primary screen of the approved drug collection and hit confirmation were deposited under assay IDs 1224859, 1224857, 1224858, 1224853, $1224855,1224861,1224862,1224860,1224856,1224854$ and 1224852.

Note: Any Supplementary Information and Source Data files are available in the online version of the paper.

\section{ACKNOWLEDGMENTS}

We thank R. Tesh and P.-Y. Shi (University of Texas Medical Branch) and the World Reference Center for Emerging Viruses and Arboviruses (WRCEVA) for the FSS-13025 isolate of ZIKV, and D. Meckes (Florida State University) for the glioblastoma SNB-19 cell line. We also thank L. Liu and Y. Cai for technical support. This work was supported by the Intramural Research Program of the National Center for Advancing Translational Sciences, National Institutes of Health (NIH) (W.Z.), ZIKV seed funding from Florida State University (H.T.), a start-up fund from Emory University (Z.W.), the Brain and Behavior Research Foundation (K.J.B.), the New York Stem Cell Foundation (K.J.B.), the Maryland Stem Cell Research Fund (G.M. and H.S.), the Simons Foundation Autism Research Initiative (award no. 308988; H.S.), and partially by NIH grants NS048271 (G.M.), NS095348 (G.M.), MH105128 (G.M.), NS047344 (H.S.), NS097206 (H.S.), MH106434 (H.S.), MH101454 (K.J.B.), MH106056 (K.J.B.), AG005138 (K.J.B.), AG046170 (K.J.B.) and AI119530 (H.T.).

\section{AUTHOR CONTRIBUTIONS}

M. Xu, E.M.L., Z.W., A.S., K.M.C., M. Xia, G.M., W.Z., H.S. and H.T. conceived of the research, designed the study, interpreted data and wrote the manuscript; $\mathrm{M}$. Xu, E.M.L., Z.W., Y.C., W.-K.H., X.Q., J.K., S.C.O., C. Hammack, F.J., H.N.N., M.I., C. Hanna, P.S., C.A., S.G.M. and W.H. performed experiments; J.T., A.G. and K.J.B. provided astrocytes; R.H. analyzed data; G.M., W.Z., H.S. and H.T. are co-senior authors who directed the research.

\section{COMPETING FINANCIAL INTERESTS}

The authors declare no competing financial interests.

Reprints and permissions information is available online at http://www.nature.com/ reprints/index.html.

1. Dick, G.W., Kitchen, S.F. \& Haddow, A.J. Zika virus. I. Isolations and serological specificity. Trans. R. Soc. Trop. Med. Hyg. 46, 509-520 (1952).

2. Heymann, D.L. et al. Zika virus and microcephaly: why is this situation a PHEIC? Lancet 387, 719-721 (2016).

3. D'Ortenzio, E. et al. Evidence of sexual transmission of Zika virus. N. Engl. J. Med. 374, 2195-2198 (2016).

4. Foy, B.D. et al. Probable non-vector-borne transmission of Zika virus, Colorado, USA. Emerg. Infect. Dis. 17, 880-882 (2011).

5. McCarthy, M. Zika virus was transmitted by sexual contact in Texas, health officials report. Br. Med. J. 352, i720 (2016).

6. Musso, D. et al. Potential sexual transmission of Zika virus. Emerg. Infect. Dis. 21, 359-361 (2015). 
7. Brasil, P. et al. Zika virus infection in pregnant women in Rio de Janeiro-preliminary report. N. Engl. J. Med. (2016). http://dx.doi.org/10.1056/NEJMoa1602412

8. Petersen, E. et al. Rapid spread of Zika virus in the Americas-implications for public health preparedness for mass gatherings at the 2016 Brazil Olympic games. Int. J. Infect. Dis. 44, 11-15 (2016).

9. Driggers, R.W. et al. Zika virus infection with prolonged maternal viremia and fetal brain abnormalities. N. Engl. J. Med. 374, 2142-2151 (2016).

10. Mlakar, J. et al. Zika virus associated with microcephaly. N. Engl. J. Med. 374, 951958 (2016).

11. Rasmussen, S.A., Jamieson, D.J., Honein, M.A. \& Petersen, L.R. Zika virus and birth defects-reviewing the evidence for causality. N. Engl. J. Med. 374, 1981-1987 (2016).

12. Cao-Lormeau, V.M. et al. Guillain-Barré syndrome outbreak associated with Zika virus infection in French Polynesia: a case-control study. Lancet 387, 1531-1539 (2016).

13. Araujo, L.M., Ferreira, M.L. \& Nascimento, O.J. Guillain-Barré syndrome associated with the Zika virus outbreak in Brazil. Arq. Neuropsiquiatr. 74, 253-255 (2016).

14. Fontes, C.A., Dos Santos, A.A. \& Marchiori, E. Magnetic resonance imaging findings in Guillain-Barré syndrome caused by Zika virus infection. Neuroradiology http://dx.doi. org/10.1007/s00234-016-1687-9 (2016).

15. Tang, H. et al. Zika virus infects human cortical neural progenitors and attenuates their growth. Cell Stem Cell 18, 587-590 (2016).

16. Garcez, P.P. et al. Zika virus impairs growth in human neurospheres and brain organoids. Science 352, 816-818 (2016)

17. Qian, X. et al. Brain-region-specific organoids using mini-bioreactors for modeling ZIKV exposure. Cell 165, 1238-1254 (2016).

18. Rossi, S.L. et al. Characterization of a novel murine model to study Zika virus. Am. J. Trop. Med. Hyg. 94, 1362-1369 (2016).

19. Lazear, H.M. et al. A mouse model of Zika virus pathogenesis. Cell Host Microbe 19, 720-730 (2016)

20. Cugola, F.R. et al. The Brazilian Zika virus strain causes birth defects in experimental models. Nature 534, 267-271 (2016).

21. Li, C. et al. Zika virus disrupts neural progenitor development and leads to microcephaly in mice. Cell Stem Cell 19, 120-126 (2016).

22. Miner, J.J. et al. Zika virus infection during pregnancy in mice causes placental damage and fetal demise. Cell 165, 1081-1091 (2016).

23. Wu, K.Y. et al. Vertical transmission of Zika virus targeting the radial glial cells affects cortex development of offspring mice. Cell Res. 26, 645-654 (2016).

24. Dang, J. et al. Zika virus depletes neural progenitors in human cerebral organoids through activation of the innate immune receptor TLR3. Cell Stem Cell 19, 258-265 (2016).

25. Larocca, R.A. et al. Vaccine protection against Zika virus from Brazil. Nature http:// dx.doi.org/10.1038/nature18952 (2016).

26. Abbink, P. et al. Protective efficacy of multiple vaccine platforms against Zika virus challenge in rhesus monkeys. Science http://dx.doi.org/10.1126/science.aah6157 (2016).

27. Yang, Y.M. et al. A small-molecule screen in stem-cell-derived motor neurons identifies a kinase inhibitor as a candidate therapeutic for ALS. Cell Stem Cell 12, 713-726 (2013).

28. Sun, W., Sanderson, P.E. \& Zheng, W. Drug combination therapy increases successful drug repositioning. Drug Discov. Today 21, 1189-1195 (2016).

29. Kouznetsova, J. et al. Identification of 53 compounds that block Ebola-virus-like particle entry via a repurposing screen of approved drugs. Emerg. Microbes Infect. 3, e84 (2014).

30. Johansen, L.M. et al. A screen of approved drugs and molecular probes identifies therapeutics with anti-Ebola virus activity. Sci. Transl. Med. 7, 290 ra89 (2015).

31. Chen, C.Z. et al. High-throughput Giardia lamblia viability assay using bioluminescent ATP content measurements. Antimicrob. Agents Chemother. 55, 667-675 (2011).

32. Debnath, A. et al. A high-throughput drug screen for Entamoeba histolytica identifies a new lead and target. Nat. Med. 18, 956-960 (2012).

33. Sun, W. et al. Chemical signatures and new drug targets for gametocytocidal drug development. Sci. Rep. 4, 3743 (2014).

34. Sun, W. et al. Rapid identification of antifungal compounds against Exserohilum ros- tratum using high-throughput drug repurposing screens. PLoS One 8, e70506 (2013).

35. He, S. et al. Repurposing of the antihistamine chlorcyclizine and related compounds for treatment of hepatitis C virus infection. Sci. Transl. Med. 7, 282 ra49 (2015).

36. Barrows, N.J. et al. A screen of FDA-approved drugs for inhibitors of Zika virus infection. Cell Host Microbe 20, 259-270 (2016).

37. Zhang, J.H., Chung, T.D. \& Oldenburg, K.R. A simple statistical parameter for use in evaluation and validation of high-throughput screening assays. J. Biomol. Screen. 4 , 67-73 (1999)

38. Hamel, R. et al. Biology of Zika virus infection in human skin cells. J. Virol. 89 8880-8896 (2015)

39. Nowakowski, T.J. et al. Expression analysis highlights $A X L$ as a candidate Zika virus entry receptor in neural stem cells. Cell Stem Cell 18, 591-596 (2016).

40. Schang, L.M., St Vincent, M.R. \& Lacasse, J.J. Five years of progress on cyclin-dependent kinases and other cellular proteins as potential targets for antiviral drugs. Antivir. Chem. Chemother. 17, 293-320 (2006).

41. Badia, R. et al. Inhibition of herpes simplex virus type 1 by the CDK6 inhibito PD-0332991 (palbociclib) through the control of SAMHD1. J. Antimicrob. Chemother. 71, 387-394 (2016)

42. Németh, G. et al. Novel, selective CDK9 inhibitors for the treatment of HIV infection. Curr. Med. Chem. 18, 342-358 (2011).

43. Okamoto, M. et al. Selective inhibition of HIV-1 replication by the CDK9 inhibitor FIT039. Antiviral Res. 123, 1-4 (2015).

44. Van Duyne, R. et al. Effect of mimetic CDK9 inhibitors on HIV-1-activated transcription. J. Mol. Biol. 425, 812-829 (2013).

45. Yamamoto, M. et al. CDK9 inhibitor FIT-039 prevents replication of multiple DNA viruses. J. Clin. Invest. 124, 3479-3488 (2014).

46. Zhang, J., Li, G. \& Ye, X. Cyclin T1-CDK9 interacts with influenza A virus polymerase and facilitates its association with cellular RNA polymerase II. J. Virol. 84, 1261912627 (2010)

47. Bell, T.M., Field, E.J. \& Narang, H.K. Zika virus infection of the central nervous system of mice. Arch. Gesamte Virusforsch. 35, 183-193 (1971)

48. Haddad, J.J. Current opinion on 3-[2-[(2-tert-butyl-phenylaminooxalyl)-amino]-propi onylamino]- 4-oxo-5-(2,3,5,6-tetrafluoro-phenoxy)-pentanoic acid, an investigational drug targeting caspases and caspase-like proteases: the clinical trials in sight and recent anti-inflammatory advances. Recent Pat. Inflamm. Allergy Drug Discov. 7 , 229-258 (2013).

49. Hoglen, N.C. et al. Characterization of IDN-6556 (3-[2-(2-tert-butyl-phenylaminooxalyl)amino]-propionylamino]-4-oxo-5-(2,3,5,6-tetrafluoro-phenoxy)-pentanoic acid): a livertargeted caspase inhibitor. J. Pharmacol. Exp. Ther. 309, 634-640 (2004).

50. Barreyro, F.J. et al. The pan-caspase inhibitor emricasan (IDN-6556) decreases liver injury and fibrosis in a murine model of non-alcoholic steatohepatitis. Liver Int. 35 953-966 (2015).

51. Shiffman, M.L. et al. Clinical trial: the efficacy and safety of oral PF-03491390, a pan-caspase inhibitor-a randomized placebo-controlled study in patients with chronic hepatitis C. Aliment. Pharmacol. Ther. 31, 969-978 (2010).

52. Pfizer, Inc. Ibrance (palbociclib) capsules (prescribing information). (Pfizer, Inc., New York, 2015).

53. Mansuy, J.M. et al. Zika virus: high infectious viral load in semen, a new sexually transmitted pathogen? Lancet Infect. Dis. 16, 405 (2016).

54. Andrews, P., Thyssen, J. \& Lorke, D. The biology and toxicology of molluscicides, Bayluscide. Pharmacol. Ther. 19, 245-295 (1982).

55. Al-Hadiya, B.M.H. Niclosamide: comprehensive profile. Profiles Drug Subst. Excip. Relat. Methodol. 32, 67-96 (2005).

56. Wu, C.J. et al. Inhibition of severe acute respiratory syndrome coronavirus replication by niclosamide. Antimicrob. Agents Chemother. 48, 2693-2696 (2004).

57. Jurgeit, A. et al. Niclosamide is a proton carrier and targets acidic endosomes with broad antiviral effects. PLoS Pathog. 8, e1002976 (2012).

58. Fang, J. et al. Identification of three antiviral inhibitors against Japanese encephalitis virus from library of pharmacologically active compounds 1,280. PLoS One 8, e78425 (2013).

59. Khanim, F.L. et al. Re-deployment-based drug screening identifies the antihelminthic niclosamide as anti-myeloma therapy that also reduces free light chain production. Blood Cancer J. 1, e39 (2011). 


\section{ONLINE METHODS}

Human forebrain-specific NPCs, astrocytes, organoids and cell line cultures. The human iPSC line (C1-2 line) was previously generated from a skin biopsy sample of a healthy male newborn and has been fully characterized and passaged on mouse embryo fibroblast (MEF) feeder layers ${ }^{60}$. The $\mathrm{BJ}$ line was derived from healthy control fibroblasts obtained from the American Type Culture Collection (ATCC) (CRL-2522, male, neonatal). All studies followed institutional review board (IRB) protocols approved by Florida State University, Johns Hopkins University School of Medicine and Icahn School of Medicine at Mount Sinai. Human iPSCs were differentiated into forebrain-specific hNPCs following the previously established protocol ${ }^{60}$. Briefly, human iPSC colonies were detached from the feeder layer with $1 \mathrm{mg} / \mathrm{ml}$ collagenase treatment for $1 \mathrm{~h}$ and suspended in embryonic body (EB) medium, consisting of fibroblast growth factor (FGF)2 -free iPSC medium supplemented with $2 \mu \mathrm{M}$ dorsomorphin (Sigma) and 2 $\mu \mathrm{M}$ A-83 (Tocris) in nontreated polystyrene plates for $4 \mathrm{~d}$ with a daily medium change. After $4 \mathrm{~d}, \mathrm{~EB}$ medium was replaced by neural induction medium (NPC medium), which consists of Dulbecco's modified Eagle's medium with F12 nutrient mixture (DMEM/F12), N2 supplement, non-essential amino acids (NEAA), $2 \mu \mathrm{g} / \mathrm{ml}$ heparin (Sigma) and $2 \mu \mathrm{M}$ cyclopamine (Tocris). Floating EBs were then transferred to Matrigel-coated 6-well plates at day 7 to form neural-tube-like rosettes. Attached rosettes were kept for $15 \mathrm{~d}$ with an NPC medium change every other day. On day 22, rosettes were picked mechanically and transferred to lowattachment plates (Corning) to form neurospheres in NPC medium containing B27 supplements (Invitrogen). Neurospheres were then dissociated with Accutase (Millipore) at $37^{\circ} \mathrm{C}$ for $10 \mathrm{~min}$ and placed onto Matrigel-coated 6-well plates at day 24 to form monolayer hNPCs in NPC medium containing B27. These hNPCs expressed forebrain-specific progenitor markers ${ }^{60}$, including NESTIN, PAX6, EMX-1, FOXG1 and OTX2.

Human BJ iPSC line and forebrain NPCs were derived as described previously $^{61,62}$. Human iPSC-derived forebrain NPCs were maintained at high density, grown on Matrigel (BD Bioscience) in medium consisting of DMEM/F12, $1 \times$ $\mathrm{N} 2,1 \times \mathrm{B} 27-\mathrm{RA}$ (Invitrogen), $1 \mu \mathrm{g} / \mathrm{ml}$ laminin (Invitrogen) and $20 \mathrm{ng} / \mathrm{ml} \mathrm{FGF-2}$ (Invitrogen). hNPCs were differentiated to astrocytes and cultured on Matrigelcoated plates in astrocyte medium (ScienCell). Astrocytes were split 1:3 every week with Accutase (Millipore) and cultured up to $120 \mathrm{~d}$.

Forebrain-specific organoids were generated from the C1-2 human iPSC line as previously described ${ }^{17}$. Briefly, on day 1 , human iPSC colonies were detached $7 \mathrm{~d}$ after passage with collagenase type IV (Thermo Fisher Scientific), washed with fresh stem cell medium and transferred to an ultra-low-attachment 6-well plate (Corning Costar), containing $3 \mathrm{ml}$ of stem cell medium (without FGF-2), plus $2 \mu \mathrm{M}$ dorsomorphine and $2 \mu \mathrm{M}$ A83-01. On days 5-6, half of the medium was replaced with induction medium consisting of DMEM/F12, $1 \times \mathrm{N} 2$ supplement (Invitrogen), $10 \mu \mathrm{g} / \mathrm{ml}$ heparin, $1 \times$ penicillin-streptomycin, $1 \times \mathrm{NEAA}, 1 \times$ Glutamax, 4 ng/ml WNT-3A (R\&D Systems), $1 \mu$ M CHIR99021 (Cellagentech) and $1 \mu \mathrm{M}$ SB-431542 (Cellagentech). On day 7, organoids were embedded in Matrigel (BD Biosciences) and continued to grow in induction medium for 6 more days. On day 14, embedded organoids were mechanically dissociated from Matrigel by pipetting up and down onto the plate with a $5-\mathrm{ml}$ pipette tip. Typically, $10-20$ organoids were transferred to each well of a 12 -well spinning bioreactor $(\operatorname{Spin} \Omega)$ containing differentiation medium, consisting of DMEM/F12, $1 \times \mathrm{N} 2$ and B27 supplements (Invitrogen), $1 \times$ penicillin-streptomycin, $1 \times 2$-mercaptoenthanol, $1 \times$ NEAA and $2.5 \mathrm{mg} / \mathrm{ml}$ insulin (Sigma). Day 18 forebrain organoids were exposed to ZIKV FSS-13025 in Spin $\Omega$ for $24 \mathrm{~h}$ and then in replaced fresh medium for an additional $9 \mathrm{~d}$ before fixation and immunostaining as previously described ${ }^{17}$. To test the potential toxic effect on hNPC proliferation, day 20 forebrain organoids were also treated with PHA-690509 $(1 \mu \mathrm{M})$ or seliciclib $(5 \mu \mathrm{M})$ for $3 \mathrm{~d}$ without ZIKV exposure. On day $23(20+3)$, treated forebrain organoids were pulsed with $10 \mu \mathrm{M}$ 5-ethynyl-2-deoxyuridine (EdU; Thermo Fisher Scientific) for $1 \mathrm{~h}$ and immediately fixed for analysis.

The glioblastoma SNB-19 cell line (part of the National Cancer Institute 60 human tumor cell lines) was a gift from Dr. David Meckes (Florida State University, Tallahassee, FL). SNB- 19 cells were maintained at $37^{\circ} \mathrm{C}$ in $5 \% \mathrm{CO}_{2}$ in RPMI-60 medium, $1 \times$ penicillin-streptomycin and 10\% FBS (Invitrogen). The Aedes albopictus $\mathrm{C} 6 / 36$ cell line (ATCC) was maintained at $28^{\circ} \mathrm{C}$ in $5 \% \mathrm{CO}_{2}$.

Preparation of ZIKV and cell infection. The MR766-ZIKV stock with the titer of $1 \times 10^{5}$ tissue-culture-infective dose (TCID)/ml, in the form of culture fluid from an infected rhesus Macaca cell line LLC-MK2, was originally obtained from ZeptoMetrix (Buffalo, NY). The FSS13025-ZIKV strain was obtained from Drs. Robert Tesh and Pei-Yong Shi (University of Texas Medical Branch, Galveston, Texas). The PRVABC59 strain was obtained from ATCC (Manassas, VA). Original viral stocks were then amplified in Aedes albopictus clone C6/36 cells. Briefly, C6/36 cells were inoculated with viral inoculum for $1 \mathrm{~h}$ at $28^{\circ} \mathrm{C}$ in a low volume of medium ( $3 \mathrm{ml}$ per T-75 flask), with rocking every $15 \mathrm{~min}$, before the addition of $17 \mathrm{ml}$ medium. Virus-inoculated cells were incubated at $28^{\circ} \mathrm{C}$ for 6-7 d before harvesting the supernatant. C6/36-amplified ZIKV titer was determined by infecting Vero cells for $48 \mathrm{~h}$ with a methylcellulose overlay and analyzed for focus-forming units per $\mathrm{ml}(\mathrm{FFU} / \mathrm{ml})$. In mock infections, an equal volume of spent uninfected C6/36 culture medium was used. For infections, cells were seeded into 12 -well plates $1 \mathrm{~d}$ before virus addition. For all cell types, compound was added $1 \mathrm{~h}$ before viral addition unless otherwise specified. Cells were harvested at $24-72 \mathrm{~h}$ after infection.

Compound libraries. The Library of Pharmacologically Active Compounds (LOPAC), which consists of 1,280 compounds, was purchased from SigmaAldrich. The NCATS pharmaceutical collection ${ }^{63}$, a collection consisting of 2,816 clinically approved and investigational drugs, was established in 2011. All compounds were dissolved in DMSO as $10-\mathrm{mM}$ stock solutions, then diluted in DMSO at a 1:3 ratio in 384-well plates, followed by reformatting into 1,536-well compound plates for use in high-throughput screening (HTS).

Caspase-3 assay. Caspase-Glo 3/7 assay kit (catalog number G8092; Promega, Madison, WI) was used to detect caspase- 3 activity. Reagents were reconstituted as described in the protocol from the manufacturer. Polystyrene plates (384-well and 1,536-well; regular tissue-culture-treated and PDL-coated) were purchased from Greiner Bio-One (Monroe, NC). Cells were seeded in 384- and 1536-well assay plates and cultured at $37^{\circ} \mathrm{C}$ with $5 \% \mathrm{CO}_{2}$ for $16 \mathrm{~h}$. ZIKV solution was added to cells, followed by incubation at $37^{\circ} \mathrm{C}$ with $5 \% \mathrm{CO}_{2}$ for $6 \mathrm{~h}$. Caspase-Glo-3/7 reagent was added to each well, unless otherwise specified, and incubated at room temperature for $30 \mathrm{~min}$. The luminescence intensity of the assay plates was measured using a ViewLux plate reader (PerkinElmer). Data were normalized by using the cell-containing wells without ZIKV as a negative control $(0 \%$ induction of caspase-3 activity) and wells containing ZIKV infected cells that induced caspase- 3 activity were used as a positive control (100\% induction of caspase 3 activity).

ATP content assay for cell viability and compound cytotoxicity. The ATPlite luminescence assay system assay kit (catalog number 6016731; PerkinElmer) was used to determine cell viability. The reagent was reconstituted and prepared as described by the manufacturer. In order to measure the cell death caused by ZIKV infection, cells were cultured for $16 \mathrm{~h}$ at $37^{\circ} \mathrm{C}$ with $5 \% \mathrm{CO}_{2}$ in assay plates, followed by addition of ZIKV solution and incubation at $37^{\circ} \mathrm{C}$ with $5 \% \mathrm{CO}_{2}$ for $72 \mathrm{~h}$. ATPlite, the ATP-monitoring reagent, was then added to the assay plates, which were then incubated for $15 \mathrm{~min}$. The resulting luminescence was measured using the ViewLux plate reader. Data were normalized using wells without cells as a control for $100 \%$ cell killing, and cell-containing wells without ZIKV infection as full cell viability ( $0 \%$ cell killing). For analysis of potential toxicity of select compounds, cells were seeded in 96-well plates. One day later, cells were treated with the indicated compounds and concentrations for $24-48 \mathrm{~h}$ before the addition of Cell Titer-Glo substrate (Promega) and measurement, according to manufacturer's instructions.

Large-scale compound screening. A quantitative high-throughput screening (qHTS, ref. 64), in which each compound was assayed in four concentrations $(0.37,1.84,9.2$ and $46 \mu \mathrm{M})$, was performed in singlet for the primary compound screen. While a single-compound concentration (in singlet) has been traditionally used for HTS of large compound collections (such as 1 to 3 million compounds), the qHTS format with multiple compound concentrations has recently been used for medium or small compound collections, such as an approved drug library. Specifically, SNB-19 cells and hNPCs were seeded onto PDL-coated 1,536-well assay plates at 250 cells per $3 \mathrm{ml}$ per well and incubated at $37^{\circ} \mathrm{C}$ in $5 \% \mathrm{CO}_{2}$ for $16 \mathrm{~h}$. Test compounds, dissolved in DMSO, were transferred to assay plates at a volume of $23 \mathrm{nl} /$ well by an automated pintool workstation (Wako Automation, San Diego, CA). Compounds were incubated with cells for $30 \mathrm{~min}$ at $37^{\circ} \mathrm{C}$ in $5 \% \mathrm{CO}_{2}$, immediately followed by the addition of $2 \mathrm{ml} /$ well of ZIKV ( $2 \mathrm{FFU} /$ cell). Incubation time 
of compound-treated cells with ZIKV varied based on assay format. Experiments measuring virus-induced caspase-3/7 activity required a 6 -h incubation of ZIKV in the presence of compounds at $37^{\circ} \mathrm{C}$ in $5 \% \mathrm{CO}_{2}$. Following this incubation, 3.5 $\mu 1 /$ well of caspase- $3 / 7$ reagent mixture was added to the assay plates. The plates were incubated for $30 \mathrm{~min}$ at room temperature, and the resultant luminescence signal was measured using a ViewLux plate reader (Perkin Elmer). Experiments measuring virus-induced cell death required a 72 - $\mathrm{h}$ incubation of ZIKV in the presence of the compounds, at $37^{\circ} \mathrm{C}$ in $5 \% \mathrm{CO}_{2}$. Following this incubation, 3.5 $\mu \mathrm{l} /$ well of ATP content detection reagent was added to assay plates. The plates were incubated for $30 \mathrm{~min}$ at room temperature, and the resultant luminescence signal was measured in a ViewLux plate reader. Step-by-step assay protocols are listed in Supplementary Tables 1 and 2.

Confirmation of primary hits and counter-screen. The primary hits were selected with criteria of $\mathrm{IC}_{50}$ values $\leq 30 \mu \mathrm{M}$, and maximal inhibition $\geq 50 \%$. The hit compounds were diluted 1:3 in DMSO to 11 concentrations for the confirmation experiments. The same caspase- 3 activity assay was first used to confirm the activity of the primary-hit compounds $(n=3)$. In order to further confirm the activity of these compounds, the protective effect of these primary hits on the cell death caused by incubation of cells with ZIKV for $3 \mathrm{~d}$ was also determined using an ATP content cell viability assay $(n=3)$.

Because compound cytotoxicity could nonspecifically reduce the caspase activity induced by ZIKV, we also used the ATP content assay to measure compound cytotoxicity in the absence of ZIKV infection. Cells were seeded in the same way as described above in 1,536-well assay plates. After a 6-h incubation with compounds in the absence of ZIKV, $3.5 \mu \mathrm{l} /$ well of ATP content reagent mixture was added to the assay plates, which were then incubated for $30 \mathrm{~min}$ at room temperature. The luminescence signal in the assay plates was measured using a ViewLux plate reader (Supplementary Table 3). Any compounds that exhibited cytotoxicity were eliminated as false-positive compounds.

Western blot analysis. Cells were harvested by trypsinization, pelleting and subsequent lysis in $1 \times$ Laemlli buffer, and the lysates were then boiled, or the cells were directly lysed in $1 \times$ Laemlli buffer and boiled. Antibodies used were anti-ZIKV NS1 (1:2,000; BF-1225-36, BioFront Technologies, Tallahassee, FL) or anti-GAPDH (sc-25778, Santa Cruz Biotechnology, Texas). The screening of two subsets of compounds for antiviral activity (Supplementary Fig. 2a and Supplementary Fig. $\mathbf{4 b}$ ) was performed in a blinded manner, whereas all other experiments were performed in a nonblinded manner.

Immunocytochemistry. Cells were fixed with $4 \%$ paraformaldehyde (Sigma) for $15 \mathrm{~min}$ at room temperature. Samples were permeabilized and blocked with $0.25 \%$ Triton X-100 (Sigma) and 10\% donkey or goat serum in phosphatebuffered saline (PBS) for 20 min as previously described ${ }^{60}$. Samples were then incubated with primary antibodies at $4{ }^{\circ} \mathrm{C}$ overnight, followed by multiple PBST (PBS plus Tween-20) washes and incubation with secondary antibodies for 1 $\mathrm{h}$ at room temperature. Slides were mounted using VECTASHIELD with 4',6diamidino-2-phenylindole (DAPI; Vectorlabs, Burlingame, CA). The following primary antibodies were used: anti-flavivirus group antigen (clone D1-4G2-415; mouse; 1:500; Millipore), anti-cleaved caspase-3 (Asp15; rabbit; 1:500; Cell Signaling Technology) and anti-phospho-histone-H3 (PH3; 9701S; rabbit; 1:300; Cell Signaling Technology) as previously described ${ }^{17}$. Antibodies were prepared in PBS containing $0.25 \%$ Triton X-100 and $10 \%$ donkey serum. For EdU assay, the Click-iT EdU Alexa Fluor 488 Imaging Kit (Thermo Fisher Scientific) was used according to the manufacturer's protocol. Images were taken by Zeiss LSM 700 and 880 confocal microscopes, an Olympus BX61 microscope or a Zeiss Axiovert 200M microscope.

For organoid studies, quantitative analyses were conducted on randomly selected cortical structures captured by confocal microscope in a blinded fashion (example as shown in Fig. 1c). Cell death and cell proliferation were quantified by counting activated caspase $-3^{+}$and $\mathrm{PH}^{+}$nuclei, respectively, relative to the total number of nuclei stained by DAPI in the ventricular structures in at least six samples, as previously described ${ }^{17}$. We determined our sample sizes based on previous studies conducted in our lab ${ }^{17}$.
NS1 enzyme-linked immunosorbent assay (ELISA). The anti-ZIKV NS1 ELISA kit (ZKV-NS1-EK) was obtained from BioFront Technologies (Tallahassee, FL) and used according to the manufacturer's protocol.

Viral titer by focus-forming unit (FFU) assay. ZIKV titers from cell supernatants were determined by infecting Vero cells for $48 \mathrm{~h}$ with a methylcellulose overlay and analyzing the plates for focus-forming units per $\mathrm{ml}$ (FFU/ml). Briefly, cell supernatant was titrated in triplicates onto a monolayer culture of Vero cells in 96-well plates and incubated at $37^{\circ} \mathrm{C}$ for $2 \mathrm{~h}$. Virus inoculum was removed and replaced with a methylcellulose overlay. Vero cells were incubated for an additional $48-72 \mathrm{~h}$ before fixation and incubated with anti-flavivirus group antigen overnight at $4{ }^{\circ} \mathrm{C}$. The next day, fixed cells were washed three times with PBS and incubated with horseradish peroxidase (HRP)-conjugated anti-mouse secondary antibody for $1 \mathrm{~h}$ at room temperature, washed again three times with PBS and incubated with DAB peroxidase substrate for $10 \mathrm{~min}$ (Vector Labs).

Antiviral compound analysis. SNB-19 cells, human astrocytes or hNPCs were seeded in 12 -well plates at approximately $3 \times 10^{5}$ cells/well. The next day, cells were treated with compound at 1 - to 10 -fold of the $\mathrm{IC}_{50}$ value, or the indicated concentration, for $1 \mathrm{~h}$ before inoculation with ZIKV (multiplicity of infection $(\mathrm{MOI})=0.5-1)$. Cells and supernatant were harvested 24-48 $\mathrm{h}$ post infection and analyzed by western blot, immunostaining or quantitative RT-PCR analyses. Western blot bands were quantified, and immunostaining images were counted using ImageJ (NIH, Bethesda, MD). For qRT-PCR analysis, total cellular RNA was purified using the RNeasy Mini Plus kit (Qiagen). About 500-1,000 ng total RNA was reverse-transcribed into cDNA using random hexamers according to manufacturer's instructions (Superscript III First Strand Synthesis System, Invitrogen). Quantitative PCR was performed using SYBR Green PCR master mix (Invitrogen), gene specific primers (ZIKV-NS1-Forward: TGGAGTTCAACTGACGGTCG; ZIKV-NS1-Reverse: TACCCCGAACCCATGATCCT; GapdhForward: TCACTGCCACCCAGAAGACTG; and Gapdh-Reverse: GGATGACCTTGCCCACAGC), and an Applied Biosystems 7500 Fast realtime PCR system. A $60{ }^{\circ} \mathrm{C}$ to $95^{\circ} \mathrm{C}$ melt curve analysis following PCR was performed using default settings. Relative quantification was performed using the $\Delta \Delta C_{\mathrm{T}}$ method with Gapdh as the endogenous control, and the relative fold change was calculated by normalizing to the levels in the control cells.

Data analysis and statistics. The primary-screen data and concentration response curves were analyzed using software developed internally ${ }^{65}$. The $\mathrm{IC}_{50}$ values of compound confirmation data were calculated using the Prism software (GraphPad Software, Inc. San Diego, CA). All values are expressed as the mean \pm s.d. unless specified otherwise. Western blots were quantified using ImageJ (NIH, Bethesda, MD). We did not exclude any samples. Experiments were not blinded, except for those that were specified. The sample sizes were estimated according to previous studies and the known variability of the assays. Statistical analyses were carried out with the one-way ANOVA using the Prism software. The $Z^{\prime}$ factor, a measure of statistical effect size and an index for assay quality control, was calculated by: $Z^{\prime}=1-\left(\left(3 \times\right.\right.$ s.d. $\left._{\text {signal }}\right)+\left(3 \times\right.$ s.d.basal $\left._{\text {. }}\right) /$ $\left(\right.$ Mean $_{\text {signal }}-$ Mean $\left.\left._{\text {basal }}\right)\right)$.

60. Wen, Z. et al. Synaptic dysregulation in a human iPS cell model of mental disorders. Nature 515, 414-418 (2014).

61. Brennand, K.J. et al. Modeling schizophrenia using human induced pluripotent stem cells. Nature 473, 221-225 (2011).

62. Topol, A. et al. Increased abundance of translation machinery in stem-cell-derived neural progenitor cells from four schizophrenia patients. Transl. Psychiatry 5, e662 (2015).

63. Huang, R. et al. The NCGC pharmaceutical collection: a comprehensive resource of clinically approved drugs enabling repurposing and chemical genomics. Sci. Transl. Med. 3, 80 ps16 (2011).

64. Inglese, J. et al. Quantitative high-throughput screening: a titration-based approach that efficiently identifies biological activities in large chemical libraries. Proc. Natl. Acad. Sci. USA 103, 11473-11478 (2006).

65. Wang, Y., Jadhav, A., Southal, N., Huang, R. \& Nguyen, D.T. A grid algorithm for high-throughput fitting of dose-response curve data. Curr. Chem. Genomics 4, 57-66 (2010). 